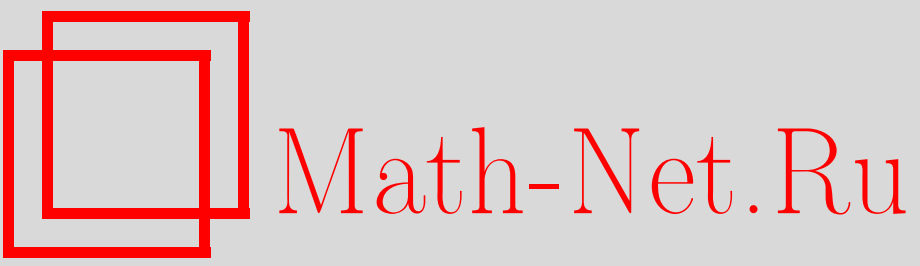

В. В. Сенатов, В. Н. Соболев, О новых формах асимптотических разложений в центральной предельной теореме, Теория вероятн. и ее примен., 2012, том 57, выпуск 1, 124-140

DOI: https://doi.org/10.4213/tvp4434

Использование Общероссийского математического портала Math-Net.Ru подразумевает, что вы прочитали и согласны с пользовательским соглашением

http: //www . mathnet.ru/rus/agreement

Параметры загрузки:

IP : 3.91 .87 .62

26 апреля 2023 г., 16:08:45 
(C) 2012 г. СЕНАТОВ В. В.* СОБОЛЕВ В. Н.**

\title{
О НОВЫХ ФОРМАХ АСИМПТОТИЧЕСКИХ РАЗЛОЖЕНИЙ В ЦЕНТРАЛЬНОЙ ПРЕДЕЛЬНОЙ ТЕОРЕМЕ ${ }^{1)}$
}

\begin{abstract}
Получены новые асимптотические разложения для плотностей в центральной предельной теореме. Приведены явные выражения для главных частей разложения Эджворта-Крамера в терминах моментов Чебышёва-Эрмита.
\end{abstract}

Ключевые слова и фразы: центральная предельная теорема, асимптотические разложения, точность аппроксимации.

Пусть $X_{1}, X_{2}, \ldots$ - независимые одинаково распределенные случайные величины с нулевым средним, единичной дисперсией и конечным моментом $\beta_{m+2}=\mathbf{E}\left|X_{1}\right|^{m+2}$, где $m$ - целое число, $m \geqslant 2$. Обозначим через $P$ общее распределение этих случайных величин и $P_{n}$ - распределение нормированной суммы $\left(X_{1}+\cdots+X_{n}\right) n^{-1 / 2}$. Нас интересуют асимптотические разложения в центральной предельной теореме (ЦПТ) для плотности $p_{n}$ распределения $P_{n}$, при этом мы предполагаем, что $\int_{-\infty}^{\infty}|f(t)|^{\nu} d t<\infty$ для некоторого положительного числа $\nu$, где $f(t)-$ характеристическая функция распределения $P$. Сходимость этого интеграла гарантирует существование непрерывной плотности $p_{n}(x)$ при всех $n \geqslant \nu$, кроме того, эту плотность можно вычислять по формуле обращения

$$
p_{n}(x)=\frac{1}{2 \pi} \int_{-\infty}^{\infty} e^{-i t x} f^{n}\left(\frac{t}{\sqrt{n}}\right) d t, \quad-\infty<x<\infty .
$$

Для построения асимптотических разложений плотности $p_{n}$ мы будем использовать моменты Чебышёва-Эрмита распределения $P$, которые определяются равенствами

$$
\theta_{k}=\theta_{k}(P)=\int_{-\infty}^{\infty} H_{k}(x) P(d x)
$$

\footnotetext{
*Московский государственный университет им. М.В.Ломоносова, механикоматематический факультет, кафедра теории вероятностей, Ленинские горы, 119991 Москва, Россия; e-mail: v.senatov@yandex.ru

** Российский государственный торгово-экономический университет, кафедра высшей и прикладной математики, ул. Смольная, 36, 125993 Москва, Россия; e-mail: sobolev_vn@mail.ru

1) Работа выполнена при поддержке РФФИ (грант № 07-01-00362).
} 
где $H_{k}(x)=(-1)^{k} \varphi^{(k)}(x) / \varphi(x), k=0,1, \ldots,-$ многочлены ЧебышёваЭрмита. Здесь и далее $\varphi(x)=e^{-x^{2} / 2} / \sqrt{2 \pi}$ - плотность стандартного нормального закона $\Phi$. При выполнении условий, наложенных на распределение $P$, величины $\theta_{k}$ существуют для всех $k \leqslant m+2$ и $\theta_{1}=\theta_{2}=0$, a $\theta_{0}=1$ для любого распределения $P$.

$\mathrm{B}$ [2] получены асимптотические разложения для плотностей $p_{n}$, $n \geqslant \max (\nu, m+1)$, в том числе разложения, называемые короткими разложениями Грама-Шарлье:

$$
\begin{aligned}
p_{n}(x)= & \varphi(x)+\sum_{l=3}^{m+1} \frac{\theta_{l}\left(P_{n}\right)}{l !} H_{l}(x) \varphi(x) \\
& +\sum_{l=m+2, l \neq 3 m-4}^{3 m-3} \frac{\theta_{l}^{(m+1)}\left(P_{n}\right)}{l !} H_{l}(x) \varphi(x)+O\left(\frac{1}{n^{m / 2}}\right)
\end{aligned}
$$

при $n \rightarrow \infty$, причем для величин $O\left(1 / n^{m / 2}\right)$ указаны явные оценки. Моменты Чебышёва-Эрмита $\theta_{l}\left(P_{n}\right)$ распределения $P_{n}$ для $l=3, \ldots, m+1$ вычисляются с помощью моментов Чебышёва-Эрмита $\theta_{3}, \ldots, \theta_{m+1}$ распределения $P$ по формулам

$$
\frac{\theta_{l}\left(P_{n}\right)}{l !}=\sum \frac{n !}{\left(n-\left(k_{3}+\cdots+k_{l}\right)\right) ! k_{3} ! \cdots k_{l} !}\left(\frac{\theta_{3}}{3 ! n^{3 / 2}}\right)^{k_{3}} \cdots\left(\frac{\theta_{l}}{l ! n^{l / 2}}\right)^{k_{l}},
$$

где суммирование ведется по всем наборам неотрицательных целых чисел $k_{3}, \ldots, k_{l}$ таким, что $3 k_{3}+\cdots+l k_{l}=l$. Известно, что $\theta_{l}\left(P_{n}\right)=O\left(1 / n^{l / 2-[l / 3]}\right)$ при $n \rightarrow \infty$. Квазимоменты $\theta_{l}^{(m+1)}\left(P_{n}\right)$, $l=m+2, \ldots, 3 m-3$, Чебышёва-Эрмита распределения $P_{n}$ вычисляются по той же формуле, в которой следует оставить только слагаемые, связанные с величинами $\theta_{j}, j \leqslant m+1$; эти квазимоменты при росте $n$ убывают так же, как и $\theta_{l}\left(P_{n}\right)$.

Суммы слагаемых в правых частях равенств вида (1), для которых указаны явные выражения, мы будем называть главными частями асимптотических разложений, а разности между $p_{n}(x)$ и главными частями разложений - остаточными частями.

Величины $\theta_{l}\left(P_{n}\right)$ и $\theta_{l}^{(m+1)}\left(P_{n}\right)$ при $l \geqslant 6$ являются суммами слагаемых, которые при росте $n$ убывают с различными скоростями (явный вид величин $\theta_{l}\left(P_{n}\right)$ для $l \leqslant 12$ указан в приложении к [2]), причём среди слагаемых, формирующих $\theta_{l}^{(m+1)}\left(P_{n}\right)$, есть слагаемые, убывающие при росте $n$ быстрее $1 / n^{m / 2}$. Например, при $m \geqslant 4$ и $l=3 m-3$ одним из наборов неотрицательных целых чисел $k_{3}, \ldots, k_{m+1}$ таких, что $3 k_{3}+\cdots+(m+1) k_{m+1}=3 m-3$, является набор, в котором $k_{3}=m-1$, а числа $k_{4}, \ldots, k_{m+1}-$ нули. Этому набору соответствует слагаемое

$$
C_{n}^{m-1}\left(\frac{\theta_{3}}{3 ! n^{3 / 2}}\right)^{m-1}=\frac{n(n-1) \cdots(n-m+2)}{(m-1) ! n^{m-1}}\left(\frac{\theta_{3}}{3 ! n^{1 / 2}}\right)^{m-1}
$$




$$
=\frac{1}{(m-1) !}\left(\frac{\theta_{3}}{3 !}\right)^{m-1} \frac{1}{n^{(m-1) / 2}}\left(1-\frac{1}{n}\right) \cdots\left(1-\frac{m-2}{n}\right),
$$

входящее в $\theta_{3 m-3}^{(m+1)}\left(P_{n}\right) /(3 m-3)$ ! и являющееся, в свою очередь, суммой $(1 /(m-1) !)\left(\theta_{3} / 3 !\right)^{m-1} / n^{(m-1) / 2}$ и слагаемых, убывающих при росте $n$ быcтреe $1 / n^{(m-1) / 2}$.

При желании мы можем записать все слагаемые из суммы по $l \geqslant m+$ 2 в правой части (1) в виде сумм величин $\left(C(P) / n^{j / 2}\right) H_{k}(x) \varphi(x), j \geqslant 2$, $k \geqslant m+2$, где числа $C(P)$ не зависят от $n$, и перенести те слагаемые, где $j \geqslant m$, из главной части разложения в остаточную часть. Так мы придем к разложению

$$
\begin{aligned}
p_{n}(x)= & \varphi(x)+\sum_{l=3}^{m+1} \frac{\theta_{l}\left(P_{n}\right)}{l !} H_{l}(x) \varphi(x) \\
& +\sum_{l=m+2, l \neq 3 m-4}^{3 m-3} \frac{\widetilde{\theta}_{l}^{(m+1)}\left(P_{n}\right)}{l !} H_{l}(x) \varphi(x)+R,
\end{aligned}
$$

где $R=O\left(1 / n^{m / 2}\right)$ при $n \rightarrow \infty$. Это разложение мы будем называть разложением Эджворта-Крамера. Представив все слагаемые из сумм по $3 \leqslant l \leqslant m+1$ и по $m+2 \leqslant l \leqslant 3 m-3, l \neq 3 m-4$, главной части разложения (2) в виде сумм величин $\left(C(P) / n^{j / 2}\right) H_{k}(x) \varphi(x), 1 \leqslant j \leqslant m-1$, $3 \leqslant k \leqslant 3 m-3, k \neq 3 m-4$, и собрав вместе все слагаемые с одним и тем же значением $j$, мы получим более привычный вид разложения Эджворта-Крамера

$$
p_{n}(x)=\varphi(x)+\sum_{j=1}^{m-1} \frac{K_{j}(x)}{n^{j / 2}} \varphi(x)+R,
$$

где $K_{j}(x)$ - линейные комбинации многочленов Чебышёва-Эрмита с коэффициентами, зависящими только от моментов распределения $P$. Во избежание недоразумений отметим, что численные значения как правых частей, так и величин $R$ в (2) и (3) совпадают. Главные части этих разложений отличаются друг от друга только способом упорядочения слагаемых. При этом стоит отметить, что один и тот же многочлен $H_{k}(x), k \geqslant 6$, участвует в формировании нескольких функций $K_{j}(x)$, $j \geqslant 2$. Например, при $m \geqslant 5$ в главную часть разложения (1) входит функция $H_{6}(x) \varphi(x)$ с коэффициентом

$$
\frac{\theta_{6}\left(P_{n}\right)}{6 !}=\frac{\theta_{6}}{6 ! n^{2}}+\frac{n-1}{2 n}\left(\frac{\theta_{3}}{3 !}\right)^{2} \frac{1}{n} .
$$

Поэтому в разложении (3) две функции, а именно $K_{2}$ и $K_{4}$, будут содержать многочлен $H_{6}$. В функцию $K_{2}$ этот многочлен входит с коэффициентом $\left(\theta_{3} / 3 !\right)^{2} / 2$, а в функцию $K_{4}-$ с коэффициентом $\theta_{6} / 6 !-\left(\theta_{3} / 3 !\right)^{2} / 2$. 
Разложение (3) является самой привычной, но одновременно и самой сложной формой записи асимптотических разложений в ЦПТ. Здесь мы имеем в виду, что если в (3) записать функции $K_{j}(x)$ в явном виде (указанном ниже в теореме 2), то получится асимптотическое разложение плотности $p_{n}(x)$, наиболее громоздкое по сравнению с другими известными разложениями.

Точность, которую гарантируют разложения (1)-(3), увеличивается (во всяком случае при больших $n$ ) при увеличении $m$ (здесь мы считаем, что распределение $P$ имеет достаточно большое число моментов). Взглянув на явный вид главных частей асимптотических разложений (хотя бы из [2]), можно заметить, что для получения все более точных разложений необходимо использовать все более сложные комбинации моментных характеристик распределения $P$. При $m=2$ главные части разложений содержат только момент $\theta_{3}$ (первые степени моментов ЧебышёваЭрмита более высоких порядков участвуют в формировании главных частей разложений при всех $m \geqslant 2$ ), при $m \geqslant 3$ главные части разложений содержат произведения пар, в том числе квадраты, моментов Чебышёва-Эрмита (при $m=3$ в главных частях появляется $\theta_{3}^{2}$ ), при $m \geqslant 4$ главные части разложений содержат произведения троек моментов и т.д. Это обстоятельство наводит на мысль о построении асимптотических разложений, в которых упорядочение слагаемых $\left(C(P) / n^{j / 2}\right) H_{k}(x) \varphi(x)$ в главной части ведется по количеству сомножителей (моментов Чебышёва-Эрмита), входящих в величины $C(P)$. Эта идея реализована в теореме 1 , формулировка и доказательство которой приведены ниже. По-видимому, форма главной части асимптотического разложения, указанная в этой теореме, является одной из простейших среди известных. С помощью теоремы 1 мы получим явные выражения многочленов $K_{j}(x)$ из (3) в терминах моментов Чебышёва-Эрмита.

Далее нам понадобятся дополнительные обозначения.

Пусть пара $(\mu, T)$, где $T>0$, а $\mu=\mu(t)$ - чётная функция, удовлетворяющая условию $e^{-t^{2} / 2} \leqslant \mu(t) \leqslant 1$ для всех действительных $t$, такова, что $|f(t)| \leqslant \mu(t)$ при $|t| \leqslant T$. О выборе конкретных пар $(\mu, T)$ см. $[2$, гл. $4, \S 6]$. Обозначим

$$
B_{k, n-1}=\frac{1}{2 \pi} \int_{-T \sqrt{n}}^{T \sqrt{n}}|t|^{k} \mu^{n-1}\left(\frac{t}{\sqrt{n}}\right) d t
$$

Нетрудно показать, что для распределения $P$ с конечным четвертым моментом пару $(\mu, T)$ можно подобрать так, что $B_{k, n-1} \rightarrow B_{k}$ при $n \rightarrow \infty$ для любого $k>0$, где $B_{k}$ - абсолютный момент порядка $k$ стандартного нормального закона, деленный на $\sqrt{2 \pi}$.

Нетрудно проверить, что моменты $\theta_{k}$ Чебышёва-Эрмита распределения $P$ и его степенные моменты $\alpha_{k}=\int_{-\infty}^{\infty} x^{k} P(d x), k \leqslant m+2$, связаны 
равенствами

$$
\frac{\theta_{k}}{k !}=\sum_{j=0}^{[k / 2]} \frac{\alpha_{k-2 j}}{(k-2 j) !} \frac{(-1)^{j}}{j ! 2^{j}} .
$$

Обозначим $\left\|\theta_{k}\right\|$ величины, определенные при четных $k$ равенством (4), в котором числа $(-1)^{j}$ заменяются на 1 , а при нечетных $k$ равенством (4), в котором, помимо этого, величины $\alpha_{k-2 j}, j \geqslant 1$, заменяются их абсолютными значениями, а $\alpha_{k}$ заменяется на $\beta_{k}$. Обозначим $\left\|\theta_{k}^{(s)}\right\|, k \leqslant m+2, s \leqslant m+1$, величины, которые определяются теми же равенствами, что и $\left\|\theta_{k}\right\|$, но в правых частях этих равенств сохраняются лишь слагаемые, связанные с моментами порядков, не больших $s$.

В $[2$, гл. $4, \S 7]$ получено следующее представление:

$$
\begin{aligned}
e^{t^{2} / 2} f(t)= & \sum_{k=0}^{m+1} \frac{\theta_{k}}{k !}(i t)^{k}+\gamma e^{t^{2} / 2} \frac{\left\|\theta_{m+2}\right\|}{(m+2) !} t^{m+2} \\
& +\gamma e^{t^{2} / 2} \frac{\left\|\theta_{m+3}^{(m+1)}\right\|}{(m+3) !} t^{m+3}, \quad-\infty<t<\infty,
\end{aligned}
$$

где $\gamma=\gamma(t)$ - непрерывные комплекснозначные функции такие, что $|\gamma| \leqslant 1$.

Следствием этого разложения являются равенства

$$
\begin{aligned}
e^{t^{2} / 2} f(t)= & \sum_{k=0}^{s} \frac{\theta_{k}}{k !}(i t)^{k}++\gamma e^{t^{2} / 2} \frac{\left\|\theta_{s+1}\right\|}{(s+1) !} t^{s+1} \\
& +\gamma e^{t^{2} / 2} \frac{\left\|\theta_{s+2}\right\|}{(s+2) !} t^{s+2}, \quad-\infty<t<\infty,
\end{aligned}
$$

для натуральных $s \leqslant m$. Функции $\gamma,|\gamma| \leqslant 1$, в различных местах работы, вообще говоря, различны.

Нам понадобятся идеальные метрики $\zeta_{s}, s>0$, введенные В. М. Золотарёвым (см., например, [1]), при этом мы будем использовать только расстояние $\zeta_{3}=\zeta_{3}(P, \Phi)$, которое можно определить как

$$
\sup \left\{\left|\int_{-\infty}^{\infty} u(x)(P(d x)-\Phi(d x))\right|: u \in \mathfrak{F}_{3}\right\},
$$

где $\mathfrak{F}_{3}$ - множество действительнозначных трижды дифференцируемых функций $u(x),-\infty<x<\infty$, таких, что $\left|u^{(3)}(x)\right| \leqslant 1$. Можно показать, что величина $\zeta_{3}$ не изменится, если вместо множества действительнозначных функций $\mathfrak{F}_{3}$ рассматривать аналогичное множество комплекснозначных функций, и почти очевидно, что для любого действительного $t$ справедливо неравенство $\left|f(t)-e^{-t^{2} / 2}\right| \leqslant|t|^{3} \zeta_{3}$. Отметим, что $\left|\theta_{3}\right| / 3$ ! $\leqslant \zeta_{3}$, причем это неравенство неулучшаемо в том смысле, что для любого числа $0<c<1$ существует распределение $P$ с нулевым средним и единичной дисперсией такое, что $\left|\theta_{3}\right| / 3 !>c \zeta_{3}$. 
Лемма. При любом $n \geqslant m+1$ для разности характеристических функиий $f^{n}(t / \sqrt{n})$ и $e^{-t^{2} / 2}$ nри $|t| \leqslant T \sqrt{n}$ справедливо представление:

$$
f^{n}\left(\frac{t}{\sqrt{n}}\right)-e^{-t^{2} / 2}=\sum_{s=1}^{m-1} C_{n}^{s} \sum_{l=3 s}^{m-1+2 s} \Theta_{s, l}\left(\frac{i t}{\sqrt{n}}\right)^{l} e^{-t^{2} / 2}+\sum_{s=1}^{m-1} r_{s}+\sum_{s=1}^{m-1} R_{s} .
$$

Здесь $\Theta_{s, l}=\sum_{t_{1}+\cdots+t_{s}=l} \theta_{t_{1}} \cdots \theta_{t_{s}} /\left(t_{1} ! \cdots t_{s} !\right)$, где суммирование ведется по наборам неотрицательных иельх чисел $t_{1}, \ldots, t_{s}$ mаким, ито $t_{1}+\cdots+$ $t_{s}=l, s=1, \ldots, m-1, l=3 s, \ldots, m-1+2 s, u t_{j} \geqslant 3, j=1, \ldots, m-1$,

$$
\begin{aligned}
&\left|r_{1}\right| \leqslant \frac{1}{n^{m / 2}}\left(\frac{\left\|\theta_{m+2}\right\|}{(m+2) !}|t|^{m+2}+\frac{\left\|\theta_{m+3}^{(m+1)}\right\|}{(m+3) ! \sqrt{n}}|t|^{m+3}\right) \mu^{n-1}\left(\frac{t}{\sqrt{n}}\right), \\
&\left|r_{s}\right| \leqslant \frac{1}{s ! n^{m / 2}} \sum_{l=3(s-1)}^{m-2+2(s-1)}\left\|\Theta_{s-1, l}\right\| \\
& \times\left(\frac{\left\|\theta_{m+2 s-l}\right\|}{(m+2 s-l) !}|t|^{m+2 s}+\frac{\left\|\theta_{m+2 s+1-l}\right\|}{(m+2 s+1-l) ! \sqrt{n}}|t|^{m+2 s+1}\right) \\
& \times \mu^{n-1}\left(\frac{t}{\sqrt{n}}\right), \quad s=2, \ldots, m-1, \\
&\left|R_{s}\right| \leqslant \frac{1}{(s+1) ! n^{m / 2}}\left\|\Theta_{s, m-1+2 s}\right\| \zeta_{3}|t|^{m+2 s+2} \mu^{n-1}\left(\frac{t}{\sqrt{n}}\right), \\
& s=1, \ldots, m-1,
\end{aligned}
$$

а величинь $\left\|\Theta_{s, l}\right\|$ определяются аналогично $\Theta_{s, l}$ с заменой величин $\theta_{t_{1}}, \ldots, \theta_{t_{s}}$ на их абсолютные значения.

Д ок а з а т ел ь с т в о. Рассмотрим разность $f^{n}(t / \sqrt{n})-e^{-t^{2} / 2}$, которую можно записать в виде $f^{n}(t / \sqrt{n})-g^{n}(t / \sqrt{n})$, где $g(t)=e^{-t^{2} / 2}$. В рассуждениях, проводимых ниже, мы - там, где это не приводит к недоразумениям - будем опускать аргументы некоторых функций, все эти аргументы суть $t / \sqrt{n}$. Из хорошо известного равенства $a^{n}-b^{n}=$ $(a-b) \sum_{j=0}^{n-1} a^{n-j-1} b^{j}$, справедливого для любых комплексных чисел $a$ и $b$, следует, что

$$
\begin{aligned}
f^{n}\left(\frac{t}{\sqrt{n}}\right)-g^{n}\left(\frac{t}{\sqrt{n}}\right) & =f^{n}-g^{n}=(f-g) \sum_{j_{1}=0}^{n-1} f^{n-j_{1}-1} g^{j_{1}} \\
& =\psi \sum_{j_{1}=0}^{n-1} f^{n-j_{1}-1} g^{j_{1}+1}
\end{aligned}
$$

где $\psi=\psi(t / \sqrt{n})=e^{t^{2} /(2 n)} f(t / \sqrt{n})-1$. Из представления функции $e^{t^{2} / 2} f(t)$, о котором говорилось выше, следует, что правая часть (7) равна

$$
\left(\sum_{t_{1}=3}^{m+1} \frac{\theta_{t_{1}}}{t_{1} !}\left(\frac{i t}{\sqrt{n}}\right)^{t_{1}}+\gamma e^{t^{2} /(2 n)} \frac{\left\|\theta_{m+2}\right\|}{(m+2) !}\left(\frac{t}{\sqrt{n}}\right)^{m+2}\right.
$$




$$
\begin{array}{r}
\left.+\gamma e^{t^{2} /(2 n)} \frac{\left\|\theta_{m+3}^{(m+1)}\right\|}{(m+3) !}\left(\frac{t}{\sqrt{n}}\right)^{m+3}\right) \sum_{j_{1}=0}^{n-1} f^{n-j_{1}-1} g^{j_{1}+1} \\
=\left(\sum_{t_{1}=3}^{m+1} \frac{\theta_{t_{1}}}{t_{1} !}\left(\frac{i t}{\sqrt{n}}\right)^{t_{1}}\right) \sum_{j_{1}=0}^{n-1} f^{n-j_{1}-1} g^{j_{1}+1}+r_{1}
\end{array}
$$

где

$$
\left|r_{1}\right| \leqslant\left(\frac{\left\|\theta_{m+2}\right\|}{(m+2) !}\left|\frac{t}{\sqrt{n}}\right|^{m+2}+\frac{\left\|\theta_{m+3}^{(m+1)}\right\|}{(m+3) !}\left|\frac{t}{\sqrt{n}}\right|^{m+3}\right) n \mu^{n-1}\left(\frac{t}{\sqrt{n}}\right) .
$$

Tak каK

$$
\begin{aligned}
\sum_{j_{1}=0}^{n-1} f^{n-j_{1}-1} g^{j_{1}+1} & =\sum_{j_{1}=0}^{n-2}\left(f^{n-j_{1}-1}-g^{n-j_{1}-1}\right) g^{j_{1}+1}+\sum_{j_{1}=0}^{n-1} g^{n} \\
& =\sum_{j_{1}=0}^{n-1} g^{n}+\sum_{j_{1}=0}^{n-2}\left((f-g) \sum_{j_{2}=0}^{n-j_{1}-2} f^{n-j_{1}-j_{2}-2} g^{j_{2}}\right) g^{j_{1}+1} \\
& =\sum_{j_{1}=0}^{n-1} g^{n}+(f-g) \sum_{j_{1}=0}^{n-2} \sum_{j_{2}=0}^{n-j_{1}-2} f^{n-j_{1}-j_{2}-2} g^{j_{1}+j_{2}+1} \\
& =\sum_{j_{1}=0}^{n-1} g^{n}+\psi \sum_{0 \leqslant j_{1}+j_{2} \leqslant n-2} f^{n-2-j_{1}-j_{2}} g^{j_{1}+j_{2}+2},
\end{aligned}
$$

TO

$$
\begin{aligned}
f^{n}-g^{n}= & \left(\sum_{t_{1}=3}^{m+1} \frac{\theta_{t_{1}}}{t_{1} !}\left(\frac{i t}{\sqrt{n}}\right)^{t_{1}}\right) \sum_{j_{1}=0}^{n-1} g^{n}+\left(\sum_{t_{1}=3}^{m} \frac{\theta_{t_{1}}}{t_{1} !}\left(\frac{i t}{\sqrt{n}}\right)^{t_{1}} \psi\right) \\
& \times \sum_{0 \leqslant j_{1}+j_{2} \leqslant n-2} f^{n-j_{1}-j_{2}-2} g^{j_{1}+j_{2}+2}+r_{1}+R_{1}
\end{aligned}
$$

где

$$
\begin{aligned}
\left|R_{1}\right| & \leqslant \frac{\left\|\Theta_{1, m+1}\right\|}{(m+1) !}\left|\frac{t}{\sqrt{n}}\right|^{m+1}\left|(f-g) \sum_{0 \leqslant j_{1}+j_{2} \leqslant n-2} f^{n-j_{1}-j_{2}-2} g^{j_{1}+j_{2}+1}\right| \\
& \leqslant \frac{\left\|\Theta_{1, m+1}\right\|}{(m+1) !}\left|\frac{t}{\sqrt{n}}\right|^{m+1} C_{n}^{2} \mu^{n-1}\left(\frac{t}{\sqrt{n}}\right) \zeta_{3}\left|\frac{t}{\sqrt{n}}\right|^{3} .
\end{aligned}
$$

Чтобы убедиться в справедливости последнего неравенства, достаточно заметить, что для любого натурального $l<n$ количество наборов неотрицательных целых чисел $j_{1}, \ldots, j_{l}$ таких, что $0 \leqslant j_{1}+\cdots+j_{l} \leqslant n-l$, равно $C_{n}^{l}$.

При $m=2$ второе слагаемое в правой части (8) следует опустить, при этом (8) принимает вид

$$
f^{n}\left(\frac{t}{\sqrt{n}}\right)-e^{-t^{2} / 2}=\frac{\theta_{3}}{3 !} \frac{(i t)^{3}}{\sqrt{n}} e^{-t^{2} / 2}+R_{1}+r_{1},
$$


где

$$
\begin{aligned}
\left|R_{1}\right| & \leqslant \frac{1}{2 n} \frac{\left\|\Theta_{1,3}\right\| \zeta_{3}}{3 !}|t|^{6} \mu^{n-1}\left(\frac{t}{\sqrt{n}}\right), \\
\left|r_{1}\right| & \leqslant \frac{1}{n}\left(\frac{\left\|\theta_{4}\right\|}{4 !}|t|^{4}+\frac{\left\|\theta_{5}^{(3)}\right\|}{5 ! \sqrt{n}}|t|^{5}\right) \mu^{n-1}\left(\frac{t}{\sqrt{n}}\right),
\end{aligned}
$$

что совпадает с утверждением леммы. Отметим, что $\left\|\Theta_{1,3}\right\|=\left|\theta_{3}\right| / 3$ !.

Далее мы считаем, что $m \geqslant 3$.

Рассмотрим сумму по $3 \leqslant t_{1} \leqslant m$ из второго слагаемого в (8). Ее можно записать в виде

$$
\begin{gathered}
\sum_{t_{1}=3}^{m} \frac{\theta_{t_{1}}}{t_{1} !}\left(\frac{i t}{\sqrt{n}}\right)^{t_{1}}\left(\sum_{t_{2}=3}^{m+3-t_{1}} \frac{\theta_{t_{2}}}{t_{2} !}\left(\frac{i t}{\sqrt{n}}\right)^{t_{2}}+\gamma e^{t^{2} /(2 n)} \frac{\left\|\theta_{m+4-t_{1}}\right\|}{\left(m+4-t_{1}\right) !}\left(\frac{t}{\sqrt{n}}\right)^{m+4-t_{1}}\right. \\
\left.+\gamma e^{t^{2} /(2 n)} \frac{\left\|\theta_{m+5-t_{1}}\right\|}{\left(m+5-t_{1}\right) !}\left(\frac{t}{\sqrt{n}}\right)^{m+5-t_{1}}\right) .
\end{gathered}
$$

Отсюда следует, что второе слагаемое в правой части (8) равно

$$
\left(\sum_{t_{1}=3}^{m} \sum_{t_{2}=3}^{m+3-t_{1}} \frac{\theta_{t_{1}} \theta_{t_{2}}}{t_{1} ! t_{2} !}\left(\frac{i t}{\sqrt{n}}\right)^{t_{1}+t_{2}}\right) \sum_{0 \leqslant j_{1}+j_{2} \leqslant n-2} f^{n-j_{1}-j_{2}-2} g^{j_{1}+j_{2}+2}+r_{2}
$$

где

$$
\begin{aligned}
\left|r_{2}\right| \leqslant & \left(\sum_{t_{1}=3}^{m} \frac{\left|\theta_{t_{1}}\right|}{t_{1} !} \frac{\left\|\theta_{m+4-t_{1}}\right\|}{\left(m+4-t_{1}\right) !}\left|\frac{t}{\sqrt{n}}\right|^{m+4}\right. \\
& \left.+\sum_{t_{1}=3}^{m} \frac{\left|\theta_{t_{1}}\right|}{t_{1} !} \frac{\| \theta_{m+5-t_{1}} \mid}{\left(m+5-t_{1}\right) !}\left|\frac{t}{\sqrt{n}}\right|^{m+5}\right) C_{n}^{2} \mu^{n-1}\left(\frac{t}{\sqrt{n}}\right) .
\end{aligned}
$$

Так мы приходим к равенству

$$
\begin{aligned}
f^{n}-g^{n}= & \left(\sum_{t_{1}=3}^{m+1} \frac{\theta_{t_{1}}}{t_{1} !}\left(\frac{i t}{\sqrt{n}}\right)^{t_{1}}\right) \sum_{j_{1}=0}^{n-1} g^{n} \\
& +\left(\sum_{6 \leqslant t_{1}+t_{2} \leqslant m+3} \frac{\theta_{t_{1}} \theta_{t_{2}}}{t_{1} ! t_{2} !}\left(\frac{i t}{\sqrt{n}}\right)^{t_{1}+t_{2}}\right) \sum_{0 \leqslant j_{1}+j_{2} \leqslant n-2} f^{n-j_{1}-j_{2}-2} g^{j_{1}+j_{2}+2} \\
& +r_{1}+r_{2}+R_{1}
\end{aligned}
$$

при этом $t_{1}, t_{2} \geqslant 3$. Далее будут появляться величины $t_{j}, j \geqslant 3$, и мы всегда считаем, что $t_{j} \geqslant 3$. При $m=3$ равенство (9) можно записать в виде

$$
\begin{aligned}
f^{n}\left(\frac{t}{\sqrt{n}}\right)-e^{-t^{2} / 2}= & \frac{\theta_{3}}{3 !} \frac{(i t)^{3}}{\sqrt{n}} e^{-t^{2} / 2}+\frac{\theta_{4}}{4 !} \frac{(i t)^{4}}{n} e^{-t^{2} / 2} \\
& +\left(\frac{\theta_{3}}{3 !}\right)^{2}\left(\frac{i t}{\sqrt{n}}\right)^{6} \sum_{0 \leqslant j_{1}+j_{2} \leqslant n-2} f^{n-j_{1}-j_{2}-2} g^{j_{1}+j_{2}+2} \\
& +r_{1}+r_{2}+R_{1} .
\end{aligned}
$$


Нетрудно проверить (см. рассуждения, приводящие к правой части (8)), что последняя сумма по $0 \leqslant j_{1}+j_{2} \leqslant n-2$ равна

$$
C_{n}^{2} g^{n}+\sum_{0 \leqslant j_{1}+j_{2}+j_{3} \leqslant n-3} f^{n-j_{1}-j_{2}-j_{3}-3} g^{j_{1}+j_{2}+j_{3}+2}(f-g) .
$$

Отсюда легко вывести, что

$$
\begin{aligned}
f^{n}\left(\frac{t}{\sqrt{n}}\right)-e^{-t^{2} / 2}= & \frac{\theta_{3}}{3 !} \frac{(i t)^{3}}{\sqrt{n}} e^{-t^{2} / 2}+\frac{\theta_{4}}{4 !} \frac{(i t)^{4}}{n} e^{-t^{2} / 2} \\
& +\frac{n-1}{2 n}\left(\frac{\theta_{3}}{3 !}\right)^{2} \frac{(i t)^{6}}{n} e^{-t^{2} / 2}+r_{1}+r_{2}+R_{1}+R_{2}
\end{aligned}
$$

где

$$
\left|R_{2}\right| \leqslant C_{n}^{3}\left(\frac{\theta_{3}}{3 !}\right)^{2} \zeta_{3} \frac{|t|^{9}}{n^{9 / 2}} \mu^{n-1}\left(\frac{t}{\sqrt{n}}\right)
$$

откуда следует утверждение леммы при $m=3$. Отметим, что $\left\|\Theta_{2,6}\right\|=$ $\left(\theta_{3} / 3 !\right)^{2}$.

Далее мы считаем, что $m \geqslant 4$. Покажем, что для любого $2 \leqslant k \leqslant$ $m-1$

$$
\begin{aligned}
f^{n}-g^{n}= & \sum_{s=1}^{k-1}\left(\sum_{l=3 s}^{m-1+2 s} \Theta_{s, l}\left(\frac{i t}{\sqrt{n}}\right)^{l}\right)_{0 \leqslant j_{1}+\cdots+j_{s} \leqslant n-s} g^{n} \\
& +\left(\sum_{l=3 k}^{m-1+2 k} \Theta_{k, l}\left(\frac{i t}{\sqrt{n}}\right)^{l}\right)_{0 \leqslant j_{1}+\cdots+j_{k} \leqslant n-k} \sum^{n-j_{1}-\cdots-j_{k}-k} g^{j_{1}+\cdots+j_{k}+k} \\
& +\sum_{s=1}^{k} r_{s}+\sum_{s=1}^{k-1} R_{s} .
\end{aligned}
$$

При $k=2$ это равенство, с точностью до обозначений, совпадает с (9). Предположим, что оно выполнено при каком-либо $2 \leqslant k<m-1$. Преобразуем $k$-е слагаемое в правой части (10), представив сумму по $0 \leqslant j_{1}+\cdots+j_{k} \leqslant n-k$ в виде

$$
\begin{aligned}
& \sum_{0 \leqslant j_{1}+\cdots+j_{k} \leqslant n-k-1}\left(f^{n-j_{1}-\cdots-j_{k}-k}-g^{n-j_{1}-\cdots-j_{k}-k}\right) g^{j_{1}+\cdots+j_{k}+k}+\sum_{0 \leqslant j_{1}+\cdots+j_{k} \leqslant n-k} g^{n} \\
= & \sum_{0 \leqslant j_{1}+\cdots+j_{k} \leqslant n-k} g^{n} \\
& +\sum_{0 \leqslant j_{1}+\cdots+j_{k} \leqslant n-k-1}\left(\sum_{j_{k+1}=0}^{n-j_{1}-\cdots-j_{k}-(k+1)} f^{n-j_{1}-\cdots-j_{k}-j_{k+1}-(k+1)} g^{j_{k+1}}(f-g)\right) \\
= & \sum_{0 \leqslant j_{1}+\cdots+j_{k} \leqslant n-k} g^{n}
\end{aligned}
$$




$$
\begin{aligned}
& +\sum_{0 \leqslant j_{1}+\cdots+j_{k+1} \leqslant n-(k+1)} f^{n-j_{1}-\cdots-j_{k+1}-(k+1)} g^{j_{1}+\cdots+j_{k+1}+k}(f-g) \\
= & \sum_{0 \leqslant j_{1}+\cdots+j_{k} \leqslant n-k} g^{n} f_{0 \leqslant j_{1}+\cdots+j_{k+1} \leqslant n-(k+1)} f^{n-j_{1}-\cdots-j_{k+1}-(k+1)} g^{j_{1}+\cdots+j_{k+1}+k+1} .
\end{aligned}
$$

Теперь нетрудно понять, что $k$-е слагаемое в правой части (10) равно

$$
\begin{aligned}
& \sum_{l=3 k}^{m-1+2 k} \Theta_{k, l}\left(\frac{i t}{\sqrt{n}}\right)^{l} \sum_{0 \leqslant j_{1}+\cdots+j_{k} \leqslant n-k} g^{n}+\left(\sum_{l=3 k}^{m-1+2 k} \Theta_{k, l}\left(\frac{i t}{\sqrt{n}}\right)^{l} \psi\right) \\
& \times \sum_{0 \leqslant j_{1}+\cdots+j_{k+1} \leqslant n-(k+1)} f^{n-j_{1}-\cdots-j_{k+1}-(k+1)} g^{j_{1}+\cdots+j_{k+1}+k+1} .
\end{aligned}
$$

Первое слагаемое в этой сумме имеет тот же вид, что и слагаемые из суммы по $1 \leqslant s<k-1$ в (10). Вторую сумму по $l$ в (12) можно записать в виде

$$
\begin{aligned}
\sum_{l=3 k}^{m-1+2 k} \sum_{t_{1}+\cdots+t_{k}=l} \frac{\theta_{t_{1}} \cdots \theta_{t_{k}}}{t_{1} ! \cdots t_{k} !}\left(\frac{i t}{\sqrt{n}}\right)^{l} \psi & \sum_{3 k \leqslant t_{1}+\cdots+t_{k} \leqslant m-1+2 k-1} \frac{\theta_{t_{1} \cdots \theta_{t_{k}}}}{t_{1} ! \cdots t_{k} !}\left(\frac{i t}{\sqrt{n}}\right)^{t_{1}+\cdots+t_{k}} \psi \\
& +\sum_{t_{1}+\cdots+t_{k}=m-1+2 k} \frac{\theta_{t_{1}} \cdots \theta_{t_{k}}}{t_{1} ! \cdots t_{k} !}\left(\frac{i t}{\sqrt{n}}\right)^{m-1+2 k} \psi
\end{aligned}
$$

Первую из сумм в правой части (13) можно записать в виде

$$
\begin{aligned}
& \quad \sum_{3 k \leqslant t_{1}+\cdots+t_{k} \leqslant m-2+2 k} \frac{\theta_{t_{1}} \cdots \theta_{t_{k}}}{t_{1} ! \cdots t_{k} !}\left(\frac{i t}{\sqrt{n}}\right)^{t_{1}+\cdots+t_{k}} \sum_{t_{k+1}=3}^{m+1+2 k-\left(t_{1}+\cdots+t_{k}\right)} \frac{\theta_{t_{k+1}}}{t_{k+1} !}\left(\frac{i t}{\sqrt{n}}\right)^{t_{k+1}} \\
& +\sum_{3 k \leqslant t_{1}+\cdots+t_{k} \leqslant m-2+2 k} \frac{\theta_{t_{1}} \cdots \theta_{t_{k}}}{t_{1} ! \cdots t_{k} !}\left(\frac{i t}{\sqrt{n}}\right)^{t_{1}+\cdots+t_{k}} \\
& \quad \times\left(\gamma e^{t^{2} /(2 n)} \frac{\left\|\theta_{m+2+2 k-\left(t_{1}+\cdots+t_{k}\right)}\right\|}{\left(m+2+2 k-\left(t_{1}+\cdots+t_{k}\right)\right) !}\left(\frac{i t}{\sqrt{n}}\right)^{m+2+2 k-\left(t_{1}+\cdots+t_{k}\right)}\right. \\
& \left.+\gamma e^{t^{2} /(2 n)} \frac{\left\|\theta_{m+3+2 k-\left(t_{1}+\cdots+t_{k}\right)}\right\|}{\left(m+3+2 k-\left(t_{1}+\cdots+t_{k}\right)\right) !}\left(\frac{i t}{\sqrt{n}}\right)^{m+3+2 k-\left(t_{1}+\cdots+t_{k}\right)}\right) .
\end{aligned}
$$

Первая из этих сумм равна

$$
\begin{gathered}
\sum_{3(k+1) \leqslant t_{1}+\cdots+t_{k+1} \leqslant m+2 k+1} \frac{\theta_{t_{1}} \cdots \theta_{t_{k+1}}}{t_{1} ! \cdots t_{k+1} !}\left(\frac{i t}{\sqrt{n}}\right)^{t_{1}+\cdots+t_{k+1}} \\
=\sum_{l=3(k+1)}^{m-1+2(k+1)} \Theta_{k+1, l}\left(\frac{i t}{\sqrt{n}}\right)^{l}
\end{gathered}
$$


а абсолютная величина второй не превосходит

$$
\begin{gathered}
e^{t^{2} /(2 n)} \sum_{l=3 k}^{m-2+2 k}\left\|\Theta_{k, l}\right\|\left(\frac{\left\|\theta_{m+2+2 k-l}\right\|}{(m+2+2 k-l) !}\left|\frac{t}{\sqrt{n}}\right|^{m+2+2 k}\right. \\
\left.+\frac{\left\|\theta_{m+3+2 k-l}\right\|}{(m+3+2 k-l) !}\left|\frac{t}{\sqrt{n}}\right|^{m+3+2 k}\right)
\end{gathered}
$$

Умножение первой из них на сумму по $0 \leqslant j_{1}+\cdots+j_{k+1} \leqslant n-(k+1)$ из (12) дает нам аналог $k$-го слагаемого из (10), в котором $k$ заменяется на $k+1$, а умножение второй на сумму по $0 \leqslant j_{1}+\cdots+j_{k+1} \leqslant n-(k+1)$ из (12) дает нам величину $r_{k+1}$, которую можно оценить произведением величины (15) без множителя $e^{t^{2} /(2 n)}$ и $C_{n}^{k+1} \mu^{n-1}(t / \sqrt{n})$.

Для завершения доказательства равенства (10), в котором $k$ заменяется на $k+1$, осталось рассмотреть величину $R_{k}$, являющуюся произведением второй суммы из правой части (13) и суммы по $0 \leqslant j_{1}+\cdots+j_{k+1} \leqslant$ $n-(k+1)$ из $(12)$. Ясно, что

$$
\left|R_{k}\right| \leqslant\left\|\Theta_{k, m-1+2 k}\right\| \zeta_{3} C_{n}^{k+1}\left|\frac{t}{\sqrt{n}}\right|^{m+2+2 k} \mu^{n-1}\left(\frac{t}{\sqrt{n}}\right) .
$$

Так мы убеждаемся в справедливости (10) при всех $2 \leqslant k \leqslant m-1$.

Положим в $(10) k=m-1$. Ясно, что при этом $(m-1)$-е слагаемое будет равно

$$
\begin{aligned}
& \Theta_{m-1,3 m-3}\left(\frac{i t}{\sqrt{n}}\right)^{3 m-3} \sum_{0 \leqslant j_{1}+\cdots+j_{m-1} \leqslant n-(m-1)} f^{n-j_{1}-\cdots-j_{m-1}-(m-1)} g^{j_{1}+\cdots+j_{m-1}+m-1} .
\end{aligned}
$$

Действуя аналогично предыдущему, легко проверить, что эта величина равна

где

$$
C_{n}^{m-1} \Theta_{m-1,3 m-3}\left(\frac{i t}{\sqrt{n}}\right)^{3 m-3} e^{-t^{2} / 2}+R_{m-1},
$$

$$
\left|R_{m-1}\right| \leqslant C_{n}^{m}\left\|\Theta_{m-1,3 m-3}\right\| \zeta_{3}\left|\frac{t}{\sqrt{n}}\right|^{3 m} \mu^{n-1}\left(\frac{t}{\sqrt{n}}\right),
$$

и доказательство леммы закончено.

В следующей теореме нам понадобятся величины

$$
L_{k}(u)=\frac{1}{\pi} \int_{u}^{\infty} t^{k} e^{-t^{2} / 2} d t, \quad u>0, \quad k=0,1, \ldots,
$$

и

$$
\alpha(u)=\max \{|f(t)|: t \geqslant u\} .
$$

Отметим, что величины $L_{k}(u)$ при $u \rightarrow \infty$ убывают экспоненциально быстро и при выполнении условия $\int_{-\infty}^{\infty}|f(t)|^{\nu} d t<\infty$ величина $\alpha(u)$ строго меньше единицы для любого $u>0$. 
Теорема 1. При выполнении условий, наложенных на распределение $P$, для любого $n \geqslant \max (\nu, m+1)$ при всех действительных $x$

$$
p_{n}(x)=\varphi(x)+\sum_{s=1}^{m-1} C_{n}^{s} \sum_{l=3 s}^{m-1+2 s} \frac{\Theta_{s, l}}{n^{l / 2}} H_{l}(x) \varphi(x)+R
$$

гдe

$$
\begin{aligned}
& |R| \leqslant \frac{1}{n^{m / 2}}\left(\frac{\left\|\theta_{m+2}\right\|}{(m+2) !} B_{m+2, n-1}+\frac{\left\|\theta_{m+3}^{(m+1)}\right\|}{(m+3) !} \frac{B_{m+3, n-1}}{\sqrt{n}}\right. \\
& +\sum_{s=2}^{m-1} \frac{1}{s !} \sum_{l=3(s-1)}^{m-4+2 s}\left\|\Theta_{s-1, l}\right\|\left(\frac{\left\|\theta_{m+2 s-l}\right\|}{(m+2 s-l) !} B_{m+2 s, n-1}\right. \\
& \left.\quad+\frac{\left\|\theta_{m+2 s+1-l}\right\|}{(m+2 s+1-l) !} \frac{B_{m+2 s+1, n-1}}{\sqrt{n}}\right) \\
& \left.+\sum_{s=1}^{m-1} \frac{1}{(s+1) !}\left\|\Theta_{s, m-1+2 s}\right\| \zeta_{3} B_{m+2+2 s, n-1}\right) \\
& +\frac{\sqrt{n}}{\pi} \alpha^{n-\nu}(T) \int_{T}^{\infty}|f(t)|^{\nu} d t+\frac{1}{T \sqrt{n}} e^{-T^{2} n / 2} \\
& +\sum_{s=1}^{m-1} C_{n}^{s} \sum_{l=3 s}^{m-1+2 s} \frac{\left|\Theta_{s, l}\right|}{n^{l / 2}} L_{l}(T \sqrt{n}) .
\end{aligned}
$$

При $m=2$ сумму по $2 \leqslant s \leqslant m-1$ в оченке величинь $R$ следует onyстить.

Д о к а з а т е л ь с т в о. Разность плотностей $p_{n}(x)$ и $\varphi(x)$ можно записать с помощью формулы обращения

$$
\begin{aligned}
p_{n}(x)-\varphi(x)= & \frac{1}{2 \pi} \int_{-\infty}^{\infty} e^{-i t x}\left(f^{n}\left(\frac{t}{\sqrt{n}}\right)-e^{-t^{2} / 2}\right) d t \\
= & \frac{1}{2 \pi} \int_{-T \sqrt{n}}^{T \sqrt{n}} e^{-i t x}\left(f^{n}\left(\frac{t}{\sqrt{n}}\right)-e^{-t^{2} / 2}\right) d t \\
& +\frac{1}{2 \pi} \int_{|t| \geqslant T \sqrt{n}} e^{-i t x} f^{n}\left(\frac{t}{\sqrt{n}}\right) d t-\frac{1}{2 \pi} \int_{|t| \geqslant T \sqrt{n}} e^{-i t x} e^{-t^{2} / 2} d t .
\end{aligned}
$$

Очевидно, что абсолютная величина последнего слагаемого не превосходит

$$
\frac{1}{\pi} \int_{-T \sqrt{n}}^{\infty} e^{-t^{2} / 2} d t \leqslant \frac{1}{\pi T \sqrt{n}} e^{-T^{2} n / 2},
$$

а абсолютная величина предпоследнего слагаемого не превосходит

$$
\frac{1}{\pi} \int_{-T \sqrt{n}}^{\infty}\left|f\left(\frac{t}{\sqrt{n}}\right)\right|^{n} d t \leq \frac{\sqrt{n}}{\pi} \alpha^{n-\nu}(T) \int_{T}^{\infty}|f(t)|^{\nu} d t
$$


и ясно, что правые части этих неравенств при росте $n$ убывают экспоненциально быстро.

Для завершения доказательства теоремы нужно воспользоваться утверждением только что доказанной леммы и при этом учесть, что

$$
\frac{1}{2 \pi} \int_{-\infty}^{\infty} e^{-i t x}(i t)^{k} e^{-t^{2} / 2} d t=H_{k}(x) \varphi(x), \quad k=0,1, \ldots
$$

И

$\frac{1}{2 \pi} \int_{-T \sqrt{n}}^{T \sqrt{n}}|t|^{k} \mu^{n-1}\left(\frac{t}{\sqrt{n}}\right) d t=B_{k, n-1}, \quad k=0,1, \ldots, \quad n \geqslant \max (\nu, m+1)$.

Теорема 1 доказана.

Далее мы будем использовать величины

$$
M_{s, l}=C_{n}^{s} \Theta_{s, l} \frac{H_{l}(x)}{n^{l / 2}},
$$

с помощью которых можно записать главную часть разложения (16) без величин $\varphi(x)$ в виде

$$
\sum_{s=1}^{m-1} \sum_{l=3 s}^{m-1+2 s} M_{s, l}
$$

Множество точек $(s, l)$, по которым производится суммирование, состоит из целочисленных точек плоскости, принадлежащих замкнутому треугольнику, стороны которого принадлежат прямым $s=1, l=3 s$, $l=m-1+2 s$. Ясно, что последняя сумма не изменится, если мы изменим порядок суммирования. Заметим, что величины $l$ на множестве суммирования изменяются от 3 до $3 m-3$. Для $l=3, \ldots, m+1$ величины $s$ изменяются от 1 до $[l / 3]$, где [·] означает целую часть, а для $l=m+2, \ldots, 3 m-3$ величины $s$ изменяются от $](l-m+1) / 2[$ до $[l / 3]$, где ] $z$ [ для положительного числа $z$ означает наименьшее целое число, большее или равное $z$. Поэтому

$$
\sum_{s=1}^{m-1} \sum_{l=3 s}^{m-1+2 s} M_{s, l}=\sum_{l=3}^{m+1} \sum_{s=1}^{[l / 3]} M_{s, l}+\sum_{l=m+2}^{3 m-3} \sum_{s=](l-m+1) / 2[}^{[l / 3]} M_{s, l} .
$$

Рассмотрим первую сумму в правой части этого равенства. Она равна

$$
\begin{aligned}
\sum_{l=3}^{m+1} \frac{H_{l}(x)}{n^{l / 2}} \sum_{s=1}^{[l / 3]} C_{n}^{s} \Theta_{s, l} \\
\quad=\sum_{l=3}^{m+1} H_{l}(x) \sum_{s=1}^{[l / 3]} C_{n}^{s} \frac{1}{n^{l / 2}} \sum_{t_{1}+\cdots+t_{s}=l} \frac{\theta_{t_{1}} \cdots \theta_{t_{s}}}{t_{1} ! \cdots t_{s} !} \\
\quad=\sum_{l=3}^{m+1} H_{l}(x) \sum_{s=1}^{[l / 3]} C_{n}^{s} \sum_{t_{1}+\cdots+t_{s}=l}\left(\frac{\theta_{t_{1}}}{t_{1} ! n^{t_{1} / 2}}\right) \cdots\left(\frac{\theta_{t_{s}}}{t_{s} ! n^{t_{s} / 2}}\right) .
\end{aligned}
$$


Зафиксируем числа $l$ и $s, 3 \leqslant l \leqslant m+1,1 \leqslant s \leqslant[l / 3]$, и рассмотрим все решения уравнения $t_{1}+\ldots+t_{s}=l$ в неотрицательных целых числах, больших или равных трем. Для каждого решения $t_{1}, \ldots, t_{s}$ обозначим $k_{3}$ число входящих в него троек, $k_{4}$ - число входящих в него четверок, ..., $k_{m+1}$ - количество входящих в него чисел $m+1$.

Ясно, что для каждого решения $t_{1}, \ldots, t_{s}$

$$
\left(\frac{\theta_{t_{1}}}{t_{1} ! n^{t_{1} / 2}}\right) \cdots\left(\frac{\theta_{t_{s}}}{t_{s} ! n^{t_{s} / 2}}\right)=\left(\frac{\theta_{3}}{3 ! n^{3 / 2}}\right)^{k_{3}} \cdots\left(\frac{\theta_{m+1}}{(m+1) ! n^{(m+1) / 2}}\right)^{k_{m+1}}
$$

при этом

$$
\begin{gathered}
3 k_{3}+\cdots+(m+1) k_{m+1}=l, \\
k_{3}+\cdots+k_{m+1}=s .
\end{gathered}
$$

Нетрудно понять, что для данного набора чисел $k_{3}, \ldots, k_{m+1}$, для которого выполнено (21), существует $C_{s}^{k_{3}} C_{s-k_{3}}^{k_{4}} \cdots C_{s-\left(k_{3}+\cdots+k_{m}\right)}^{k_{m+1}}$ решений уравнения $t_{1}+\cdots+t_{s}=l$ с заданными числами $k_{3}, \ldots, k_{m+1}\left(k_{3}\right.$ троек можно разместить на $s$ позициях в сумме $t_{1}+\cdots+t_{s}$ числом способов, равным $C_{s}^{k_{3}}, k_{4}$ четверок можно поместить на $s-k_{3}$ оставшихся позициях $C_{s-k_{3}}^{k_{4}}$ способами и т.д.). Поэтому последняя сумма по $s=1, \ldots,[l / 3]$ из (19) равна

$$
\begin{aligned}
\sum_{s=1}^{[l / 3]} C_{n}^{s} & \sum^{\prime} C_{s}^{k_{3}} C_{s-k_{3}}^{k_{4}} \cdots C_{s-\left(k_{3}+\cdots+k_{m}\right)}^{k_{m+1}} \\
& \times\left(\frac{\theta_{3}}{3 ! n^{3 / 2}}\right)^{k_{3}} \cdots\left(\frac{\theta_{m+1}}{(m+1) ! n^{(m+1) / 2}}\right)^{k_{m+1}}
\end{aligned}
$$

где сумма $\sum^{\prime}$ берется по всем наборам неотрицательных целых чисел $k_{3}, \ldots, k_{m+1}$, для которых выполнены условия $(20)$ и $(21)$. Легко проверить, что сумма (22) равна

$$
\begin{aligned}
& \sum_{s=1}^{[l / 3]} \sum^{\prime} \frac{n !}{\left(n-\left(k_{3}+\cdots+k_{m+1}\right)\right) ! k_{3} ! \cdots k_{m+1} !} \\
& \quad \times\left(\frac{\theta_{3}}{3 ! n^{3 / 2}}\right)^{k_{3}} \cdots\left(\frac{\theta_{m+1}}{(m+1) ! n^{(m+1) / 2}}\right)^{k_{m+1}} .
\end{aligned}
$$

Очевидно, что для $l \leqslant m+1$ все величины $k_{j}$ из (20) с $j \geqslant l+$ 1 с необходимостью равны нулю и поэтому условия (20) и (21) можно записать в виде

$$
\begin{gathered}
3 k_{3}+\cdots+l k_{l}=l, \\
k_{3}+\cdots+k_{l}=s .
\end{gathered}
$$


Из первого из этих равенств следует, что $3 k_{3}+\cdots+3 k_{l} \leqslant l$, поэтому заведомо $s \leqslant[l / 3]$, т.е. сумма $(22)$ равна

$$
\sum_{s \geqslant 1} \sum^{\prime \prime} \frac{n !}{\left(n-\left(k_{3}+\cdots+k_{l}\right)\right) ! k_{3} ! \cdots k_{l} !}\left(\frac{\theta_{3}}{3 ! n^{3 / 2}}\right)^{k_{3}} \cdots\left(\frac{\theta_{l}}{l ! n^{l / 2}}\right)^{k_{l}},
$$

где сумма $\sum^{\prime \prime}$ берется по наборам неотрицательных целых чисел $k_{3}, \ldots, k_{l}$, для которых выполнены условия (23) и (24). Легко понять, что сумма (25) не изменится, если мы снимем в ней суммирование по $s \geqslant 1$, исключив из ограничений условие (24). Отсюда следует, что первая из сумм в правой части (18), умноженная на $\varphi(x)$, совпадает с суммой по $3 \leqslant l \leqslant m+1$ из правой части $(1)$.

Рассмотрим сумму по $s$ во втором слагаемом в правой части (18). Рассуждения, аналогичные только что проведенным, приводят к формуле, аналогичной $(25)$, в которой $k_{l}$ заменяется на $k_{m+1}$, последний сомножитель заменяется на $\left(\theta_{m+1} /\left((m+1) ! n^{(m+1) / 2}\right)\right)^{k_{m+1}}$, суммирование в $\sum^{\prime \prime}$ ведется по наборам неотрицательных целых чисел, для которых выполняются условия (20) и (21), а суммирование во внешней сумме в $(25)$ ведется по целым $s \geqslant(l-m+1) / 2$.

Таким образом, главную часть разложения (16) можно записать в виде, аналогичном виду главной части разложения (1) с тем лишь отличием, ито в коэффиииентах при $H_{l}(x) \varphi(x), l \geqslant m+2$, участвуют аналоги квазимоментов $\theta_{l}^{(m+1)}\left(P_{n}\right)$ Чебышёва-Эрмита, при определении которых налагается дополнительное условие

$$
k_{3}+\cdots+k_{m+1} \geqslant \frac{l-m+1}{2} .
$$

Можно сказать, что это разложение находится между разложениями (1) и (2), поскольку если его записать в виде суммы величин $\left(C(P) / n^{j / 2}\right) H_{k}(x) \varphi(x), j \geqslant 1,3 \leqslant k \leqslant 3 m-3$, где $C(P)$ не зависят от $n$, то среди этих слагаемых будут слагаемые с $j \geqslant m$, а с другой стороны, количество указанных слагаемых будет меньше, чем в разложении (1). Так, в этом разложении коэффициентом при $H_{3 m-3}(x) \varphi(x)$ является величина $C_{n}^{m-1}\left(\theta_{3} / 3 !\right)^{m-1} / n^{(3 m-3) / 2}=O\left(1 / n^{(m-1) / 2}\right)$ при $n \rightarrow \infty$, а коэффициент при $H_{3 m-3}(x) \varphi(x)$ в разложении (1) содержит при $m \geqslant 5$, помимо только что указанной величины, слагаемые

$$
\begin{aligned}
C_{n}^{1} C_{n-1}^{m-3} \frac{\theta_{6}}{6 ! n^{3}}\left(\frac{\theta_{3}}{3 ! n^{3 / 2}}\right)^{m-3}=O\left(\frac{1}{n^{(m+1) / 2}}\right), \quad n \rightarrow \infty, \\
C_{n}^{3}\left(\frac{\theta_{m-1}}{(m-1) ! n^{(m-1) / 2}}\right)^{3}=O\left(\frac{1}{n^{3(m-3) / 2}}\right), \quad n \rightarrow \infty .
\end{aligned}
$$

Образно говоря, слагаемые, связанные с величинами (27) и (28), в главную часть (18) не пропускает условие (26). Отметим, что в (18) не участвует многочлен Чебышёва-Эрмита порядка $3 m-4$. 
З а м е ч а н и е. В [2] были получены разложения вида (1), в которых участвовали как квазимоменты $\theta_{l}^{(m+1)}\left(P_{n}\right)$, так и квазимоменты $\theta_{l}^{(m)}\left(P_{n}\right)$, определяемые аналогично. В частности, именно последние квазимоменты использовались в теореме 4 главы 4 , в которой $m=5$. Этим обусловлено отсутствие слагаемого (27), т.е. $C_{n}^{1} C_{n-1}^{2}\left(\theta_{6} /\left(6 ! n^{3}\right)\right)\left(\theta_{3} /\left(3 ! n^{3 / 2}\right)\right)^{2}$, в коэффициенте при $H_{12}(x) \varphi(x)$ в утверждении этой теоремы.

Получим еще одну форму главной части разложения (16) без величин $\varphi$, т.е. для суммы (17).

Так как каждая целочисленная точка $(s, l)$ плоскости принадлежит некоторой прямой $l=j+2 s$ и $j=0, \pm 1, \ldots$, то сумма $(17)$ не изменится, если мы вначале просуммируем числа $M_{s, l}$ по парам $(s, l)$, лежащим на данной прямой $l=j+2 s$, где $j$ таково, что прямая $l=j+2 s$ пересекается с указанным выше треугольником, а затем просуммируем полученные числа по всем таким $j$.

Нетрудно понять, что самая верхняя из интересующих нас прямых есть $l=m-1+2 s$, а самая нижняя есть $l=1+2 s$ (она пересечется с упомянутым выше треугольником в единственной точке $(1,3))$. Теперь почти очевидно, что сумма (17) равна

$$
\sum_{j=1}^{m-1} \sum_{s=1}^{j} M_{s, l}=\sum_{j=1}^{m-1} \sum_{s=1}^{j} C_{n}^{s} \frac{\Theta_{s, j+2 s}}{n^{j / 2+s}} H_{j+2 s}(x) .
$$

Поэтому разложению (16) можно придать вид

$$
p_{n}(x)=\varphi(x)+\sum_{j=1}^{m-1} \frac{1}{n^{j / 2}} \sum_{s=1}^{j} \frac{C_{n}^{s}}{n^{s}} \Theta_{s, j+2 s} H_{j+2 s}(x) \varphi(x)+R,
$$

где величина $R-$ та же, что и в утверждении теоремь 1.

Отметим, что $C_{n}^{s} / n^{s} \rightarrow 1 / s$ ! при $n \rightarrow \infty$.

Преобразуем часть разложения (29) без величин $\varphi(x)$ с тем, чтобы получить в общем случае явный вид многочленов $K_{j}(x), j=1, \ldots, m-1$, участвующих в разложении Эджворта-Крамера (3).

Очевидно, что величина $C_{n}^{s}$ является многочленом $s$-го порядка переменной $n$, т.е.

$$
C_{n}^{s}=\sum_{r=1}^{s} b_{s, r} n^{r}
$$

где $b_{s, s}=1 / s !, b_{s, s-1}=-1 /(2(s-2) !), \ldots, b_{s, 1}=(-1)^{s-1} / s$.

Поэтому сумму $1 \leqslant j \leqslant m-1$ из правой части (29) можно записать в виде

$$
\sum_{j=1}^{m-1} \frac{1}{n^{j / 2}} \sum_{s=1}^{j}\left(\sum_{r=1}^{s} b_{s, r} \frac{1}{n^{s-r}}\right) \Theta_{s, j+2 s} H_{j+2 s}(x) \varphi(x) .
$$


Ясно, что величина $K_{j}(x) / n^{j / 2}, j=1, \ldots, m-1$, является частью суммы

$$
\sum_{k=1}^{j} \frac{1}{n^{k / 2}} \sum_{s=1}^{k}\left(\sum_{r=1}^{s} b_{s, r} \frac{1}{n^{s-r}}\right) \Theta_{s, k+2 s} H_{k+2 s}(x),
$$

причем суммирование во внешней сумме достаточно проводить по $k$ той же четности, что и $j$.

Положив $k=j-2 d, d=0,1, \ldots,[j / 2]$, мы можем предыдущее выражение записать в виде

$$
\frac{1}{n^{j / 2}} \sum_{d=0}^{[j / 2]} n^{d} \sum_{s=1}^{j-2 d}\left(\sum_{r=1}^{s} b_{s, r} \frac{1}{n^{s-r}}\right) \Theta_{s, j-2 d+2 s} H_{j-2 d+2 s}(x),
$$

при этом мы считаем, что сумма пустого множества слагаемых равна нулю. Теперь понятно, что

$$
K_{j}(x)=\sum_{d=0}^{[j / 2]} \sum_{s=1}^{j-2 d} b_{s, s-d} \Theta_{s, j-2 d+2 s} H_{j-2 d+2 s}(x),
$$

при этом $s-d \geqslant 1$. Поэтому

$$
K_{j}(x)=\sum_{d=0}^{[j / 2]} \sum_{s=1+d}^{j-2 d} b_{s, s-d} \Theta_{s, j-2 d+2 s} H_{j-2 d+2 s}(x) .
$$

Внутренняя сумма в этом выражении содержит непустое множество слагаемых лишь при $1+d \leqslant j-2 d$, т.е. при $d \leqslant(j-1) / 3$. Так как $(j-1) / 3 \leqslant[j / 2]$ при всех $j \geqslant 1$, то мы получаем следующее утверждение.

Теорема 2. Многочлень $K_{j}(x), j=1,2, \ldots, m-1$, допускают следующее представление:

$$
K_{j}(x)=\sum_{d=0}^{[(j-1) / 3]} \sum_{s=1+d}^{j-2 d} b_{s, s-d} \Theta_{s, j+2 s-2 d} H_{j+2 s-2 d}(x) .
$$

В заключение отметим, что величины, которые формируются примерно так же, как $\Theta_{s, l}$, использовались в работе [3].

\section{СПИСОК ЛИТЕРАТУРЫ}

1. Золотарёв В. М. Современная теория суммирования независимых случайных величин. М.: Наука, 1986, 415 с.

2. Сенатов В. В. Центральная предельная теорема: Точность аппроксимации и асимптотические разложения. М.: Книжный дом «Либроком», 2009, 352 с.

3. Сюлюкин A. B. Об асимптотических разложениях Бергстрема-Чебышёва. - Теория вероятн. и ее примен., 2009, т. 54, № 1, с. 176-185.

Поступила в редакцию 29.X.2009 